\title{
Role of susceptibility weighted imaging in acute ischemic stroke
}

\author{
${ }^{1}$ Tarek Ibrahiem Menecie, ${ }^{2}$ Sherif Abd-El-fattah Khedr, ${ }^{1}$ Mohammad Ali Saeed Hassan, \\ ${ }^{1}$ Mahmoud Hassan Mohammad Zaghloul \\ ${ }^{1}$ Department of Neuorology, Faculty of Medicine- Al-Azhar University, ${ }^{2}$ Department of Radiology, \\ Faculty of Medicine, Cairo University \\ Corresponding author: Mahmoud Hassan Mohammad Zaghloul, Mobile: 01013904246; Email: mahhas290@ gmail.com
}

\begin{abstract}
Background: stroke is a clinical syndrome of rapidly developing symptoms and signs of focal loss of cerebral function without apparent cause other than vascular origin, lasting 24 hours at least, or may lead to death. Objective: in our study, we aimed to determine the diagnostic value of susceptibility weighted image in detection of cerebral micro bleeds and prediction of hemorrhagic transformation in patients with acute ischemic stroke and their impact on choosing the most appropriate therapeutic protocol. Patients and Methods: this study included 28 patients with acute ischemic stroke. Patients admitted in Neurology Department of Nasser Institute Hospital within $72 \mathrm{~h}$ after the onset of the neurological symptoms, during the study period from $1 / 1 / 2018$ to 30/8/2018, their age ranged between 23-85 years. Results: SWI image was a highly sensitive MRI sequence for detection and evaluation of cerebral micro bleeds especially on 3T magnet. Microbleeds were traditionally categorized according to their presumed underlying etiology into lobar (CAA related pathology) and deep (arteriosclerosis) microbleeds. There was a highly significant positive correlation between both age and hypertention with number of both cortical and basal ganglionic microbleeds. While, the age was obviously more correlated with cotical microbleeds, hypertention, in contrast, was more correlated with basal ganglionic microbleeds. Conclusion: presence of microbleeds in cerebral infarct lesions requires a comprehensive assessment for the therapeutic option, especially when using thrombolytic therapy or anticoagulants, but a relatively small number of microbleeds would not affect safety when using antiplatelet therapy.
\end{abstract}

Keywords: hemorrhagic transformation, diffusion-weighted imaging, susceptibility-weighted imaging

\section{INTRODUCTION}

Stroke is a clinical syndrome of rapidly developing symptoms and signs of focal loss of cerebral function without apparent cause other than vascular origin, lasting 24 hours at least, or may lead to death before this ${ }^{(1)}$.Worldwide, stroke is the second leading cause of mortality, resulting around $6,000,000$ deaths per year ${ }^{(2)}$.Strokes can be classified into two major types: ischemic and hemorrhagic. Ischemic strokes are due to interrupted brain tissue blood supply while hemorrhagic strokes are caused by extravasation of blood out of a blood vessel or an abnormal vascular structure. About $85 \%$ of strokes are ischemic and the rest are hemorrhagic. Some hemorrhages develop inside areas of ischemia (Hemorrhagic transformation). Actually, it is unknown how many hemorrhagic strokes start as ischemic stroke ${ }^{(3)}$.Understanding of the pathogenesis of brain damage following ischemia is to understand how ischemia occurs. Ischemia is a reduction in blood flow sufficient to interfere with normal cellular function. Brain tissue is highly sensitive to ischemia, so even brief ischemic periods to neurons can lead to a complex sequence of events that may culminate in cellular death ${ }^{(2)}$.SWI is a highly sensitive in detecting hemorrhage so allows good visualization of the hemorrhagic lesion. Thromboembolism can also change susceptibility by decreasing arterial blood flow, thus increasing deoxyhemoglobin levels, and may also increase pooling of deoxygenated blood ${ }^{(4)}$.

Several important tissues have unique magnetic susceptibility differences relative to surrounding tissues. Signals from these substances are out of phase with background tissues at sufficiently long echo times. Thus phase imaging is a good means for enhancing contrast in magnetic resonance (MR) imaging. In 1997, it was possible to remove most of the unwanted phase artifacts and keep the local phase of interest ${ }^{(5)}$. Combination of the phase and the magnitude information made a new susceptibility weighted magnitude image, which is called nowadays as 
susceptibility-weighted imaging (SWI) ${ }^{(6)}$. Although SWI depends on GE sequences, it has more enhanced susceptibility sensitivity compared to conventional $\mathrm{T}^{*}$ weighted GE sequences because it is based on a highresolution, long TE, flow-compensated, 3D GE imaging technique that contains filtered phase information in each voxel ${ }^{(7)}$.Recently, SWI has been shown to detect $67 \%$ more CMBs than a conventional GRE $\mathrm{T}_{2}{ }^{*}$ sequence ${ }^{(8)}$.It can detect micro- and macrohemorhages and delineate cerebral microvasculature and can also discover low-flow vascular malformations. It has been proven as a complementary, valuable imaging sequence in management of stroke patients. It differentiates between calcium and hemorrhage in the brain ${ }^{(9)}$.SWI can also detect spontaneous hemorrhagic transformation of ischemic stroke earlier than CT scans ${ }^{(\mathbf{1 0})}$.Despite its efficacy in improving clinical outcome of acute ischemic stroke patients, application of thrombolytic therapy with tissue plasminogen activator (tPA) or by mechanical thrombectomy is limited by strict guidelines, due to increased risk of hemorrhagic transformation (HT) after the 4.5 or 6-h therapeutic time windows of thrombolysis or thrombectomy, respectively. Nevertheless, some patients may still benefit from thrombolysis even after 4.5-6 h of stroke onset ${ }^{(11)}$.In acute ischemic stroke, presence of prominent veins, microbleeds and the "susceptibility vessel sign" in SWI has been considered to be useful in evaluating stroke severity, treatment and outcome (12).Also, cerebral microbleeds are a radiological biomarker of the cerebral small vessel diseases that are liable for bleeding and cause most of spontaneous intracerebral hemorrhages ${ }^{(13)}$.

\section{AIM of the WORK}

This study was designed to determine the diagnostic value of susceptibility weighted imaging in detection of cerebral microbleeds and prediction of hemorrhagic transformation in patients with acute ischemic stroke and their impact on choosing the most appropriate therapeutic protocol.

\section{PATIENTS and METHODS}

This study was consisted of 28 subjects including patients with acute ischemic stroke, Patients admitted in Neurology Department of Nasser Institute Hospital within $72 \mathrm{~h}$ after the onset of the neurological symptoms, during the study period from $1 / 1 / 2018$ to $30 / 8 / 2018$, their age ranged between 23-85 years. This study was approved by the ethical committee of the Faculty of Medicine, Al-Azhar University,Cairo.

\section{The patients were selected according to the following criteria:}

Inclusion criteria: patients with acute ischemic stroke who presented within 72 hours of neurological symptom onset with clinical and radiological confirmation.

Exclusion criteria: patients with initial brain computed tomography (CT) showed an evidence of an intracranial hemorrhage, either (subdural, extradural, intracerebral, subarachnoid hemorrhage). Other stroke mimics included: transient ischemic attack, brain tumor and multiple sclerosis, patients with contraindications for MRI and patients who refused to participate in this study.

Ethical considerations: all steps of the study had been explained clearly to all the patients and /or family before doing the procedure.

\section{Statistical Analysis:}

Data were coded, computed then analyzed using IBM SPSS (Statistical package for social science) version 24 for windows to obtain:

Descriptive statistics: qualitative data: were presented by frequency tables (Number and percentages).

Quantitative variables: the normality of data was first tested with Shapiro-Wilk test and presented data by central indices and dispersion: Mean \pm Standard deviation (SD) for normally distributed variables. Median and range (Minimum Maximum) for non-normally distributed variables.

Analytical statistics: Chi-square test is used to test association between categorical variables. It is replaced by Fisher Exact Test if the expected cell count was less than 5 in four-cells tables, while is replaced by Monte Carlo test if the expected cell count was less than 5 in more than four-cells tables. Association between normally distributed continuous variables was tested using independent sample t-test. Mann-Whitney U test (z) was used to compare non-parametric continuous variables Spearman correlation used to correlate ordinal and non-normally distributed data. 
Level of significance: for all above mentioned statistical tests the results were considered RESULTS significant when the probability of error is less than or equal $5 \%(\mathrm{p} \leq 0.05)$.

Table 1: demographic characters and basic clinical data (risk factors) of the studied group $(\mathrm{N}=28)$

\begin{tabular}{|l|ll|c|}
\hline \multicolumn{1}{|c|}{ Parameters } & \multicolumn{1}{c|}{ Items } & N (\%) \\
\hline Age & $($ Mean \pm SD) & $35.11 \pm 13.8$ \\
\multirow{2}{*}{ Gender } & $>$ & Male & $17(60.7 \%)$ \\
& $>$ & Female & $11(39.3 \%)$ \\
\hline \multirow{2}{*}{ Smoking } & $>$ & Yes & $15(53.6 \%)$ \\
& $>$ & No & $13(46.4 \%)$ \\
\hline \multirow{2}{*}{ DM } & $>$ & Yes & $13(46.4 \%)$ \\
& $>$ & No & $15(53.6 \%)$ \\
\hline \multirow{2}{*}{ HTN } & $>$ & Yes & $21(75 \%)$ \\
\hline \multirow{2}{*}{ IHD } & $>$ & No & $7(25 \%)$ \\
\hline \multirow{2}{*}{ AF } & $>$ & Yes & $10(35.7 \%)$ \\
\hline \multirow{2}{*}{ EF $\mathbf{5 0}$} & $>$ & No & $18(64.3 \%)$ \\
\hline \multirow{2}{*}{ SWMA } & $>$ & Yes & $4(14.3 \%)$ \\
\hline \multirow{2}{*}{ LAD } & $>$ & No & $24(85.7 \%)$ \\
\hline \multirow{2}{*}{ Previous CVS } & $>$ & Yes & $3(10.7 \%)$ \\
\hline
\end{tabular}

Data expressed as mean \pm SD or number $\%$.

HTN: hypertension, DM: diabetes Mellitus, IHD: ischemic heart disease, AF: atrial fibrillation, EF: ejection fraction, SWMA: segmental wall motion abnormality, LAD: left atrial dilatation, CVS: cerebrovascular stroke and SD: standard deviation.

Table 2: spearman correlation between age and cortical microbleeds and BG microbleeds

\begin{tabular}{|c|c|c|}
\hline Items & r & P value \\
\hline Age - cortical microbleeds & 0.66 & $\leq \mathbf{0 . 0 0 1 *}$ \\
\hline Age - BG microbleeds & 0.57 & $\mathbf{0 . 0 0 1} *$ \\
\hline
\end{tabular}

*: significant $\mathrm{p} \leq 0.05$.

r: correlation coefficient.

Table 3: relation between hypertension and cortical and BG microbleeds

\begin{tabular}{|c|c|c|c|c|}
\hline \multirow[b]{2}{*}{ Items } & \multicolumn{2}{|c|}{ Hypertension } & \multirow[t]{2}{*}{ Test of significance } & \multirow[b]{2}{*}{$P$ value } \\
\hline & $\begin{array}{c}\text { Yes } \\
n=21\end{array}$ & $\begin{array}{l}\text { No } \\
n=7\end{array}$ & & \\
\hline Cortical microbleeds & $1(0-4)$ & $0(0-1)$ & $\begin{array}{c}\text { MW } \\
Z=2.5\end{array}$ & $0.01 *$ \\
\hline BG microbleeds & $1(0-7)$ & $0(0-1)$ & $\begin{array}{c}\text { MW } \\
Z=2.7\end{array}$ & $0.006 *$ \\
\hline
\end{tabular}

Table 4: spearman correlation between stroke severity (admission NIHSS), stroke outcome (discharge MRS) and infarction volume

\begin{tabular}{|c|c|c|}
\hline Items & R & P value \\
\hline Admission NIHSS - Discharge mRS & 0.92 & $\leq \mathbf{0 . 0 0 1}$ \\
\hline Admission NIHSS - Infarction volume & 0.51 & $\mathbf{0 . 0 0 5}^{*}$ \\
\hline Discharge mRS - Infarction volume & 0.62 & $\leq \mathbf{0 . 0 0 1}$ \\
\hline
\end{tabular}

\footnotetext{
$*$ : significant $\mathrm{p} \leq 0.05$.
}

r: correlation coefficient 
Table 5: relation between hemorrhagic transformation and presence of microbleeds

\begin{tabular}{|c|c|c|c|}
\hline \multirow[b]{2}{*}{ Items } & \multicolumn{2}{|c|}{ HT } & \multirow[t]{2}{*}{$P$ value } \\
\hline & $\begin{array}{l}\text { Yes } \\
n=4\end{array}$ & $\begin{array}{c}\text { No } \\
n=24\end{array}$ & \\
\hline $\begin{array}{ll}\text { Cortical microbleeds } \\
> & \text { Yes } \\
> & \text { No }\end{array}$ & $\begin{array}{l}2(50 \%) \\
2(50 \%)\end{array}$ & $\begin{array}{l}14(58.3 \%) \\
10(41.7 \%)\end{array}$ & $\begin{array}{c}\text { FET } \\
\mathrm{P}=0.99\end{array}$ \\
\hline $\begin{array}{l}\text { BG microbleeds } \\
>\quad \text { Yes } \\
>\quad \text { No } \\
\end{array}$ & $\begin{array}{l}3(75 \%) \\
1(25 \%) \\
\end{array}$ & $\begin{array}{l}14(58.3 \%) \\
10(41.7 \%)\end{array}$ & $\begin{array}{c}\text { FET } \\
\mathrm{P}=0.93\end{array}$ \\
\hline \begin{tabular}{ll}
\multicolumn{3}{l}{ Microbleeds } \\
$>$ & Yes \\
$>$ & No \\
$>$
\end{tabular} & $\begin{array}{l}3(75 \%) \\
1(25 \%)\end{array}$ & $\begin{array}{c}17(70.8 \%) \\
7(29.2 \%)\end{array}$ & $\begin{array}{c}\text { FET } \\
\mathrm{P}=0.94\end{array}$ \\
\hline
\end{tabular}

FET: Fisher's Exact Test. Not significant $\mathrm{p}>0.05$

Table 6: relation between occurrence of hemorrhagic transformation (HT) and severity of stroke and outcome

\begin{tabular}{|c|c|c|c|}
\hline \multirow[b]{2}{*}{ Items } & \multicolumn{2}{|c|}{ HT } & \multirow[t]{2}{*}{ Test of sig } \\
\hline & $\begin{array}{l}\text { Yes } \\
n=4\end{array}$ & $\begin{array}{c}\text { No } \\
n=24\end{array}$ & \\
\hline $\begin{array}{ll}\text { Severity on admission (NIHSS) } \\
> & \text { Mild } \\
> & \text { Moderate } \\
> & \text { Sever }\end{array}$ & $\begin{array}{c}0(0 \%) \\
2(50 \%) \\
2(50 \%)\end{array}$ & $\begin{array}{c}9(37.5 \%) \\
13(54.2 \%) \\
2(8.3 \%)\end{array}$ & $\begin{array}{l}\mathrm{MC}=5.68 \\
\mathbf{P}=\mathbf{0 . 0 5 *}\end{array}$ \\
\hline $\begin{array}{ll}\text { Outcome (discharge MRS) } \\
> & \text { Favorable } \\
> & \text { Unfavorable and death }\end{array}$ & $\begin{array}{c}0(0 \%) \\
4(100 \%)\end{array}$ & $\begin{array}{c}19(79.2 \%) \\
5(20.8 \%)\end{array}$ & $\begin{array}{c}\text { FET } \\
\mathbf{P}=\mathbf{0 . 0 0 6}^{*}\end{array}$ \\
\hline
\end{tabular}

FET: Fisher's Exact Test

MC: Monte Carlo Test

*: significant $\mathrm{p} \leq 0.05$

\section{DISCUSSION}

Cerebral microbleeds (CMBs) are small foci of chronic blood products in normal or near normal brain tissue, with a diameter of less than about 5 $\mathrm{mm}{ }^{(14)}$. They can be detected by paramagnetic sensitive MR sequences such as T2* weighted gradient recalled echo (GRE) or susceptibility weighted sequences. They are generally not seen on CT, or on FLAIR, T1 weighted or T2 weighted sequences ${ }^{(15)}$. In our study, we aimed at presenting the role of susceptibility weighted image in evaluation and prognosis of acute ischemic stroke by studying role of microbleeds as a predictor of ischemic stroke outcome.SWI detected old micro-bleeds in twenty patients out of 28 (71\%), while CT could not detect any of these micro-bleeds. This was in agreement with ${ }^{(12)}$ and ${ }^{(4)}$ who reported that SWI, with its higher sensitivity, detects numerous microbleeds unidentified on any of routine images. Results of Charidimou et al. (16) supported the idea that CMBs in ischemic stroke patients were a radiological marker for more severe and potentially progressive small vessel pathology, that require active treatment including good control of BP and other vascular risk factors. Koennecke ${ }^{(17)}$ reported that the prevalence of CMBs among patients with ischemic stroke varies widely (0 to 78\%). A study of Kidwell et al. ${ }^{(18)}$ reported prevalence ranged from $12 \%$ to $71 \%$. Our study is consistent with that and showed a high prevalence for microbleeds (71\%), cotical microbleeds were found in about (57\%) and basal ganglionic microbleeds in about $(61 \%)$ of patients. This result may be attributed to high sensitivity of SWI image for microbleeds over conventional $\mathrm{T}_{2} *$ image especially on 3 Tesla magnet. Cordonnier et al. ${ }^{(\mathbf{1 9})}$ stated that in people with cerebrovascular diseases BMB prevalence varied from $41.6 \%$ when using a 1.0 Tmagnet to $46.9 \%$ when using a $1.5 \mathrm{~T}$ magnet and Nandigam et al. ${ }^{\left({ }^{(8)}\right.}$ stated that SWI has recently been shown to detect $67 \%$ more CMBs than conventional GRE $\mathrm{T}_{2} *$ sequence. It is also consistent with high prevalence of hypertention among our patients. In the present study, there were a relatively low 
numbers (0-4) for cortical MBs and 0-7 for basal ganglionic MBs. Patients used different treatments, 12 patients received only aspirin, while 9 patients received aspirin and clopidogrel, 3 patients received aspirin and anticoagulant ( 1 of them developed HT), 1 patient received aspirin and rtpa (developed HT), 1 patient received dual antiplatelet and anticoagulant (developed HT), 1 patient received anticoagulant only (developed HT) and 1 patient received no treatment. Patients on anticoagulant were significantly more associated with BG MBs (not cortical MBs) than patients on antiplatelets only. Akoudad et al. ${ }^{(20)}$ stated that coumarin users had a higher prevalence of deep or infratentorial microbleeds and a higher incidence of any microbleeds. In our study, incidence of HT was about $14 \%$ of patients, this is in agree with a controlled clinical trial in acute ischemic stroke reported the rates of total HT over the first 5 days from $3.2 \%$ to $37 \%$ in the placebo group and from $10.6 \%$ to $44 \%$ in the group who received thrombolytic treatment, meanwhile, symptomatic HT ranged from $0.6 \%$ to $7 \%$ in the placebo group and from $6.4 \%$ to $20 \%$ in the thrombolyzed group ${ }^{(21)}$.In our study all patients with hemorrhagic transformation (4 patients $=14 \%$ ) were with large infarctions (120-192-220-264) $\mathrm{cm} 3$ and their NIHSS was 18-15-17-15 respectively. Three of them were with high SBP (130-220-200-190) respectively. Two of them were AF patients with cardiomyopathy. All those patients were received medical supportive measurements in ICU. Management was planned for each patient according to the presence or absence of hemorrhagic transformation of infarction or associated old microbleeds. The presence of microbleeds or hemorrhagic transformation of infarctions limited, sometimes, the use of conventional dose of dual anti-platelets and postponed using of anticoagulants (when indicated) and also required restricted management of high blood pressure aiming to avoid serious complications. Our results are in agreement with those of Wycliffe et al. ${ }^{(22)}$ and Santhosh et al. ${ }^{(12)}$; their results indicated that SWI was useful in the evaluation of acute stroke patients being highly sensitive to even tiny hemorrhages and has potential diagnostic, as well as therapeutic, implications in acute stroke patients, and this technique should be included in the routine evaluation of stroke patients, specially where it is essential to rule out presence of hemorrhage within the infarct with a high degree of accuracy as thrombolysis and even full dose of anticoagulants in such patients can be catastrophic.

In agree with results of Cordonnier et al. ${ }^{(19)}$ our study revealed that both cortical and BG microbleeds were significantly associated with IHD, but not with gender, smoking. BG MBs were associated with DM and cortical MBs were not.

In our study, as regarding hemorrhagic transformation, there was no significant relation between hemorrhagic transformation and presence of these small numbers of microbleeds. These results are in agreement with those of Koennecke ${ }^{(17)}$ who did not provide an evidence that $\mathrm{CMB}$ increase the risk of ICH among patients on antithrombotic treatment or those treated with thrombolysis for acute stroke.

Kidwell et al. (18) found no increase in hemorrhagic transformation following tPA treatment, while Nighoghossian et al. (10) suggested that CMBs (detected on MRI after treatment was initiated) increased the risk of future hemorrhagic transformation of cerebral infarction or ICH. In our study, regarding stroke severity (evaluated by NIHSS) and its outcome (measured by $\mathrm{mRS}$ ), there was a significant positive relation between hemorrhagic transformation and the stroke severity and its outcome.

Recent population-based data supported the possibility that CMB location (deep hemispheric or infratentorial versus strictly lobar) which might relate to specific underlying vascular pathology. Analysis of the APOE genotype in individuals from the Rotterdam Scan Study grouped by CMB distribution showed an association of the APOE $\varepsilon 4$ allelle with isolated lobar CMBs, but not with deep hemispheric or infratentorial CMBs Vernooij et al. (23) Given the relation between APOE $\varepsilon 4$ and cerebral amyloid angiopathy, these results raised the possibility that individuals with isolated lobar CMBs (58\% of CMB-positive people in the Rotterdam study) might have advanced cerebral amyloid angiopathy. In the same study, systolic blood pressure, severe hypertension, and lacunar infarcts were more closely associated with deep or infratentorial CMBs, suggesting hypertensive vasculopathy as the predominant underlying 
pathology in this subgroup Chalmers et al. ${ }^{(24)}$ Of course, our study had some limitations because the baseline backgrounds of the patients were not necessarily the same and because the number of patients was small, some results may be controversial and may not be confirmed in studies with a larger number of patients. In addition, the present study was an observational study and causality has yet to be established because bias and confounding could not be eliminated in an observational study.

\section{CONCLUSION}

SWI image was a highly sensitive MRI sequence for detection and evaluation of cerebral microbleeds especially on 3T magnet. There was a highly significant positive correlation between both age and hypertention with number of both cortical and basal ganglionic microbleeds. And while the age was obviously more correlated with cotical microbleeds, hypertention, in contrast, was more correlated with basal ganglionic microbleeds. Presence of microbleeds in cerebral infarct lesions requires a comprehensive assessment for the therapeutic option, CMBs may prevent thrombolysis and anticoagulation especially with large infarct volume while antiplatelet therapy is more safer therapeutic option.

\section{REFERENCES}

1. World Health Organization (2010): Stepwise approach to stroke surveillance. Cited from: Menicie, T., Zayed, A., Hablas, W., Shahin, E., Zaki, M., \& Mahmoud, M. (2017). Prognosis of Patients with Acute Ischemic Stroke through Clinical and Magnetic Resonance Image Studies Al-Azhar Medical Journal, 331(4146), 1-7.

2. Woodruff T, Thundyil J, Tang S et al. (2011): Pathophysiology, treatment and animal and cellular models of human ischemic stroke. Molecular Neurodegeneration, 1(2): 6-11.

3. Donnan GA, Fisher M, Macleod M et al. (2008): Stroke. Lancet, 371:1612-1623.

4. Mittal $S, W u ~ Z$, Neelavalli $J$ et al. (2009). Susceptibility-weighted imaging: technical aspects and clinical applications, part 2. American Journal of Neuroradiology, 30(2): 232252.

5. Reichenbach JR, Venkatesan R, Schillinger DJ et al. (1997): Small vessels in the human brain: MR venography with deoxyhemoglobin as an intrinsic contrast agent. Radiology, 204:272-277.

6. Haacke EM, Mittal $S, W u ~ Z$ et al. (2009). Susceptibility-weighted imaging: technical aspects and clinical application. American Journal of Neuroradiology, 30(1): 19-30.

7. Gasparotti $R$, Pinelli $L$ and Liserre $R$ (2011): New MR sequences in daily practice: susceptibility weighted imaging. A pictorial essay. Insights Imaging, 2:335-347.

8. Nandigam RN, Viswanathan A, Delgado P et al. (2009): MR imaging detection of cerebral microbleeds: effect of susceptibility-weighted imaging, section thickness, and field strength. American Journal of Neuroradiology, 30(2): 338-343.

9. Halefoglu AM and Yousem DM (2018): Susceptibility weighted imaging: Clinical applications and future directions. World Journal of Radiology, 10(4): 30-39.

10. Nighoghossian $\mathbf{N}$, Hermier $M$, Adeleine P et al. (2002): Old microbleeds are a potential risk factor for cerebral bleeding after ischemic stroke: a gradient-echo $\mathrm{T} 2 *$-weighted brain MRI study. Stroke, 33(3): 735-742.

11. Mokin M, Rojas H and Levy EI (2016): Randomized trials of endovascular therapy for stroke impact on stroke care. Nature Reviews Neurology, 12(2): 86-94.

12. Santhosh $K$ and Kesavadas $C$, Thomas $B$ et al. (2009): Susceptibility weighted imaging: a new tool in magnetic resonance imaging of stroke. Clin. Radiol., 64:74-83.

13. Wardlaw JM, Smith EE, Biessels GJ et al. (2013): Neuroimaging standards for research into small vessel disease and its contribution to ageing and neurodegeneration. The Lancet Neurology, 12(8): 822-838.

14. Greenberg SM, Vernooij MW, Cordonnier C et al. (2009): Cerebral microbleeds: a guide to detection and interpretation. The Lancet Neurology, 8(2): 165-174.

15. Shoamanesh A, Kwok CS and Benavente O (2011): Cerebral microbleeds: histopathological correlation of neuroimaging. Cerebrovascular Diseases, 32(6): 528-534.

16. Charidimou A, Peeters A, Fox $\mathrm{Z}$ et al. (2012): Spectrum of transient focal neurological 
episodes in cerebral amyloid angiopathy: multicentre magnetic resonance imaging cohort study and meta-analysis. Stroke, 43(9): 23242330.

17. Koennecke HC (2006): Cerebral microbleeds on MRI: prevalence, associations, and potential clinical implications. Neurology, 66(2): 165-171.

18. Kidwell CS, Saver JL, Carneado J et al. (2002): Predictors of hemorrhagic transformation in patients receiving intra-arterial thrombolysis. Stroke, 33(3):717-724.

19. Cordonnier C, Al-Shahi Salman R and Wardlaw J (2007): Spontaneous brain microbleeds: systematic review, subgroup analyses and standards for study design and reporting. Brain, 130(8): 1988-2003.

20. Akoudad S, Darweesh SK, Leening MJ et al. (2014): Use of coumarin anticoagulants and cerebral microbleeds in the general population. Stroke, 45:3436-3439.
21. Zhang J, Yang Y, Sun H and Xing Y (2014): Hemorrhagic transformation after cerebral infarction: current concepts and challenges. Annals of Translational Medicine, 2:8.

22. Wycliffe ND, Choe J, Holshouser B et al. (2004): Reliability in detection of hemorrhage in acute stroke by a new three dimensional gradient recalled echo susceptibility weighted imaging technique compared to computed tomography: a retrospective study. Journal of the International Society for Magnetic Resonance in Medicine, 20(3): 372-377.

23. Vernooij $M$ W, van der Lugt A, Ikram M, Wielopolski P A, Niessen W, Hofman A and Breteler M (2008): Prevalence and risk factors of cerebral microbleeds The Rotterdam Scan Study. Neurology, 70(14), 1208-1214.

24. Chalmers K, Wilcock $\mathbf{G} K$ and Love $S$ (2003): APOE 4 influences the pathological phenotype of Alzheimer's disease by favouring cerebrovascular over parenchymal accumulation of $A \beta$ protein. Neuropathology and Applied Neurobiology, 29(3), 231-238. 